\title{
Quantitatively characterizing sandy soil structure altered by microbial-induced carbonate precipitation \MICPУ using multi-level thresholding segmentation on synchrotron radiation imaging
}

Tao Liu

Nanjing University

Wei Zhang ( $\nabla$ wzhang@nju.edu.cn )

Nanjing University https://orcid.org/0000-0002-6853-9518

Xiaohua Pan

Nanjing University

Jingtao Wang

Nanjing University

Lu Deng

Nanjing University

Hong-hu Zhu

Nanjing University

\section{Research Article}

Keywords: Soil structure, Micro-CT, Multi-level thresholding, Genetic algorithm, Microbially induced carbonate precipitation

Posted Date: September 9th, 2021

DOl: https://doi.org/10.21203/rs.3.rs-804069/v1

License: (c) (i) This work is licensed under a Creative Commons Attribution 4.0 International License. Read Full License 


\section{Quantitatively characterizing sandy soil structure altered by}

\section{2 microbial-induced carbonate precipitation (MICP) using multi-level}

3 thresholding segmentation on synchrotron radiation imaging

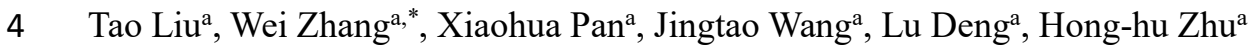

Abstract: The influences of biological, chemical, and flow processes on soil structure through microbial-induced carbonate precipitation (MICP) are not yet fully understood. In this study, we use the Kapur entropy (KE) multi-level thresholding segmentation algorithm to quantitatively characterize sandy soil structure altered by MICP treatment. A sandy soil specimen was treated by MICP and scanned by the synchrotron radiation micro-CT with a resolution of $6.5 \mu \mathrm{m}$. After validation, tri-level thresholding segmentation using KE successfully separated the precipitated calcium carbonate crystals from sand particles and pores. Spatial distribution of porosity, pore structure parameters, and flow characteristics were calculated for quantitative characterization. The size effect of the specimen was discovered to be a key factor affecting the performance of MICP treated soil. The results offer pore-scale insights of MICP treatment effect, and the quantitative understanding confirms the importance of the KE multi-level thresholding segmentation algorithm.

Keywords: Soil structure; Micro-CT; Multi-level thresholding; Genetic algorithm; Microbially induced carbonate precipitation

\section{Introduction}

Microbial-induced carbonate precipitation (MICP) is a novel environment-friendly and sustainable soil stabilization technique (Dejong et al., 2013; Shashank et al., 2016). It utilizes the natural bio-geochemical reactions to result in inorganic cementation between soil grains, which improves the engineering properties of soils, e.g., permeability, bearing capacity, liquefaction resistance and erosion resistance (Frankel \& Bazylinski, 2003; Whiffin et al., 2007; Dejong et al., 2010; Liu et al., 2020).

The joint effect of the size, shape, and arrangement of soil particles and the interaction between them is collectively referred to as soil structure, and the engineering properties of soils depend on 
the soil structure (Mitchell and Soga, 2005). During the process of MICP, the carbonate precipitation within the pore space volume altered the former soil structure. But how the biological, chemical, and flow process of MICP influence soil structure is still not fully understood (Jiang et al., 2020).

Micro-computed tomography (micro-CT), with a cross section pixel size in the micrometer range, is an efficient tool to reveal the minute details of soil structure owing to its non-invasive fluoroscopy and high spatial resolution (Oda et al., 2004; Sleutel et al., 2008; Hasan \& Alshibli, 2010). Advanced micro-CT imaging techniques based on synchrotron radiation further enhance the spatial resolution and avoid common artifacts (Schropp et al., 2012) to reveal more minute details of soil structures (Voltolini et al., 2017). In addition, the spatial structures of soils can be characterized by three-dimensional reconstruction ( $\mathrm{Li}$ et al., 2017). However, the quantitative characterization results largely rely on subjective operator judgements for factors such as sample differences, equipment parameter adjustments, and noise. This is especially true when performing image segmentation to separate different substances. For the grayscale images of the soil obtained by micro-CT scanning, the watershed algorithm (Taylor et al., 2015; Zheng \& Hryciw., 2016) and the single thresholding (Sezgin et al., 2004) methods can be used for binary segmentation to separate the particles from the pores. However, as for the soil treated by MICP, binary segmentation is incapable of differentiating the precipitated calcium carbonate crystals from the soil particles and pores in that three greyscales exist (Wang et al., 2011; Houston et al., 2013; Wei et al., 2019). Therefore, previous characterization methods can only provide the general morphological description of the soil structure of MICP treated soils; but the extent to which the soil structure and flow characteristics are altered by MICP remains unclear (Dejong et al., 2013). Obviously, developing more reliable indicators is needed (Iaasonov et al., 2009; Baveye et al., 2010; Cnudde \& Boone, 2013).

Multi-level thresholding subdivides different objects in the image by setting multiple thresholds (Para et al., 2016). It holds much potential in segmenting the soils treated by MICP, as multiple thresholds distinguishing different types of particles can be identified. The selection criteria of multi-level thresholding based on the division of histogram regions, such as the Otsu (Otsu, 1979) and Kapur entropy algorithms (Kapur et al., 1985), can be used to determine multiple thresholds (Bhandari et al., 2016). Previous studies indicated that, when applied to soil images of micro-CT, Otsu thresholding might lead to unreliable results (Houston et al., 2013). Moreover, studies have 
confirmed the advantages of Kapur entropy in multi-level thresholding segmentation; this is because no prior knowledge is required, and small objects in the image can be retained (Wu et al., 2015; Pare et al., 2016). However, few works on segmenting the MICP treated soil using multi-level thresholding has been implemented.

In this study, grayscale image sequences of an MICP-treated quartz silty sand column were obtained using synchrotron radiation micro-CT. A novel multi-level thresholding algorithm was proposed to identify the thresholds for directly separating calcium carbonate crystals from sand particles and pores. The applicability and accuracy of the algorithm were validated by four indices. Quantitative comparisons of soil structures and flow characteristics between representative element volumes with and without calcium carbonate crystals were performed using the maximal ball algorithm (Dong \& Blunt, 2009; Arand \& Hesser, 2017) and the lattice Boltzmann method (Chen \& Doolen, 1998; Guo et al., 2020). Micro-scale insights of MICP treatment effect were gained from the study; reversely, the quantitative understanding confirms the importance of the KE multi-level thresholding segmentation algorithm.

\section{Algorithm principle}

Kapur entropy (Kapur et al., 1985) (KE) considers the gray-scale probability of the pixels in a digital image and maximizes the amount of information in the image after segmentation. The entropy value is calculated in accordance with the probability in the gray level class (i.e., the proportion of the pixel amount at a certain gray level to the total pixel amount in the class); therefore, the size of the target and its background are not sensitive enough to retain the small target in the image (Wu et al., 2015). In terms of Shannon entropy, the histogram entropy $H$ of a grayscale image is defined as:

$$
H=-\sum_{i=0}^{l-1} p_{i} \ln p_{i}
$$

where $l$ is the gray level of a digital image, and $p_{i}$ is the probability of the $i$ th gray level. For a digital image with an 8-bit depth, the value of $l$ is $256\left(2^{8}\right)$.

Assume a binary threshold $t$ segments the digital image as group object (O) and group background (B), the discrete probability distributions of $\mathrm{O}$ and $\mathrm{B}$ yield: 


$$
\left\{\frac{p_{0}}{\sum_{i=0}^{t} p_{i}}, \frac{p_{1}}{\sum_{i=0}^{t} p_{i}}, \ldots, \frac{p_{t}}{\sum_{i=0}^{t} p_{i}}\right\}
$$

86

$$
H(t)=H_{O}(t)+H_{B}(t)=\frac{H+\sum_{i=0}^{t} p_{i} \ln p_{i}}{1-\sum_{i=0}^{t} p_{i}}-\frac{\sum_{i=0}^{t} p_{i} \ln p_{i}}{\sum_{i=0}^{t} p_{i}}+\ln \sum_{i=0}^{t} p_{i}\left(1-\sum_{i=0}^{t} p_{i}\right)
$$

Based on the definition in Eq. (1), the entropies of the object and background are $H_{O}(t)$ and $H_{B}(t)$, respectively, which yields:

$$
H_{B}(t)=-\sum_{i=t+1}^{l-1} \frac{p_{i}}{1-\sum_{i=0}^{t} p_{i}} \ln \frac{p_{i}}{1-\sum_{i=0}^{t} p_{i}}=\ln \left(1-\sum_{i=0}^{t} p_{i}\right)+\frac{H+\sum_{i=0}^{t} p_{i} \ln p_{i}}{1-\sum_{i=0}^{t} p_{i}}
$$

Thus, the total entropy of the image, $H(t)$, yields:

An optimal threshold value $t^{*}$ exists that maximizes $H(t)$, which yields:

$$
t^{*}=\arg \max _{0 \leq t \leq l-1} H(t)
$$

When extended to multi-level thresholding, assume that $n$ thresholds $t_{1}, t_{2}, \mathrm{~K}$, $t_{\mathrm{n}}$ exist, which satisfies $t_{1}<t_{2}<\mathrm{L}<t_{n}$. The total entropy yields:

$$
H\left(t_{1}, t_{2}, \ldots, \mathrm{t}_{n}\right)=\ln \left(\sum_{i=0}^{t_{1}} p_{i}\right)+\ln \left(\sum_{i=t_{1}+1}^{t_{2}} p_{i}\right)+\mathrm{L}+\ln \left(\sum_{i=t_{n}}^{l-1} p_{i}\right)-\frac{\sum_{i=0}^{\mathrm{t}_{1}} p_{i} \ln p_{i}}{\sum_{i=0}^{t} p_{i}}-\frac{\sum_{i=t_{1}+1}^{\mathrm{t}_{2}} p_{i} \ln p_{i}}{\sum_{i=s_{1}+1}^{t_{2}} p_{i}}-\mathrm{L}-\frac{\sum_{i=t_{n}+1}^{l-1} p_{i} \ln p_{i}}{\sum_{i=t_{n}+1}^{l-1} p_{i}}
$$


99 An assembly of optimal thresholds $\left\{t_{1}^{*}, t_{2}^{*}, \ldots, t_{\mathrm{n}}^{*}\right\}$ exists that maximizes the total entropy, that is

$$
\left\{t_{1}^{*}, t_{2}^{*}, \ldots, t_{\mathrm{n}}^{*}\right\}=\arg \max _{0 \leq t_{1}<t_{2}<\ldots \triangleleft_{n} \leq l-1} H\left(t_{1}, t_{2}, \ldots, t_{n}\right)
$$

101

102

103

104

105

106

107

108

109

110

111

112

113

114

115

116

117

118

119

120

121

122

123

124

125

\section{Material and methods}

\subsection{MICP treatment}

A soil sample treated by MICP was prepared in the laboratory. Quartz sand C190 Accusand (70-120 mesh, Unimin Corporation, Le-Sueur, USA) with a specific gravity of 2.65 and porosity of 0.699 was used. Considering the high-precision imaging and subsequent test results for processing, the particle size distribution primarily ranges from 0.15 to $0.21 \mathrm{~mm}$. The bacterial species (Sporosarcina pasteurii, ATCC 11859) was offered by the China General Microbiological Culture Collection Center. The turbidimetric method was used to detect biomass in the configured bacterial solution (Sutton, 2011). At a wavelength of $600 \mathrm{~nm}$, the characterization of turbidity (OD600) was 0.63. A cementation solution consisting of $1 \mathrm{~mol} / \mathrm{L}$ urea and $1 \mathrm{~mol} / \mathrm{L} \mathrm{CaCl}_{2}$ was used to provide chemicals that induce calcite precipitation during treatment.

Figure 1 sketches the configuration of the MICP cementation test. A grouting liquid container, a peristaltic pump, a sand column sample, and a waste liquid container were used for MICP cementation. Specifically, the peristaltic pump injects the grouting liquid, controlling the grouting speed and volume. A sand column mold, made of round transparent plexiglass pipe with an inner diameter of $10 \mathrm{~mm}$ and height of $100 \mathrm{~mm}$, contains the sand column. The preparation of such a small specimen is for the accuracy of the micro-CT scanning. The sample preparation process was executed as follows. First, the bottom of the column mold was covered with a layer of rapid qualitative filter paper; the side hose was filled with a piece of gauze to avoid leakage of sand particles. During the sample preparation, water was added, and sand was gradually added to the mold by occasionally tapping the side wall to allow the sand particles to sink freely, avoiding stratification during sedimentation. As the height of the sand column reached about $50 \mathrm{~mm}$, the addition of sand was halted, and the remaining space of the mold was filled with pieces of gauze to prevent the emergence of sand during grouting. Finally, the bottom and top threads of the mold were tightened with raw tape to avoid water leakage. 


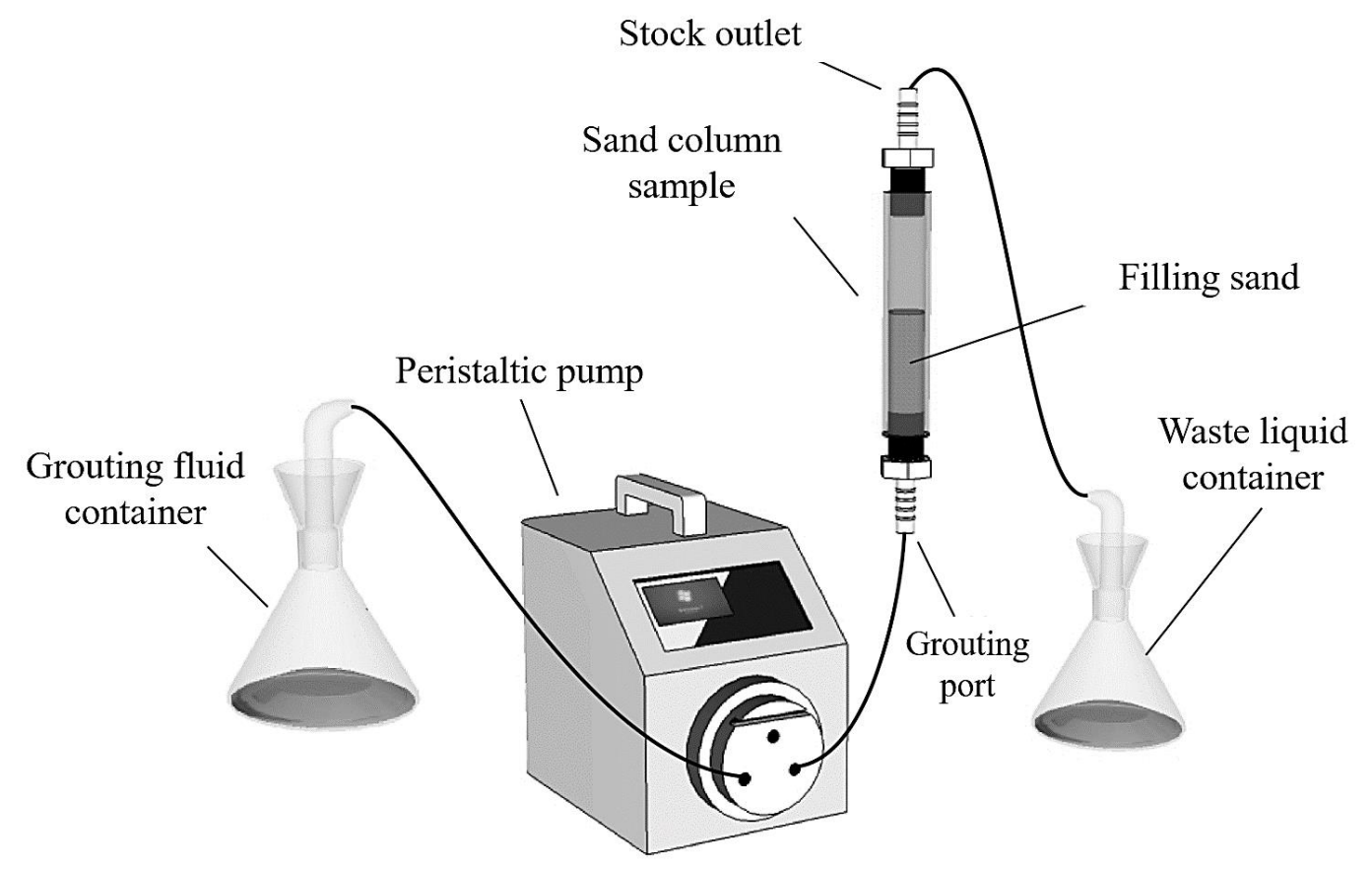

Fig. 1 Sketched configuration of MICP cementation

Grouting was performed at a constant temperature of $30{ }^{\circ} \mathrm{C}$. The bacterial liquid was first injected from the grouting port at a rate of $0.2 \mathrm{ml} / \mathrm{min}$ and a pressure of $0.2 \mathrm{bar}$, until the turbid and yellow bacterial liquid flowed out of the stock outlet. After standing for $2 \mathrm{~h}$, the cementation solution was injected at a rate of $0.02 \mathrm{ml} / \mathrm{min}$ for $40 \mathrm{~h}$; then, a round of grouting batch was completed. Only two grouting batches were conducted, because the amount of carbonate precipitation is deliberately controlled for the accuracy validation of the KE multi-level algorithm. The permeability coefficient was measured before and after each grouting batch. To ensure the specimen was not disturbed, sample preparation and MICP treatment were continuously performed in situ before micro-CT scanning. For convenience, the sample was defined as an "initial sample" and a "cemented sample" before and after MICP cementation, respectively.

The sand column is a one-dimensional seepage system that follows Darcy's law. The permeability coefficient was measured by a constant head permeability test. Table 1 lists the measured flow rates and permeability coefficients. After two rounds of grouting, the flow rate across the sample decreased from 3.7 to $1.05 \mathrm{~mL} / \mathrm{min}$ (71.62\%), while the permeability coefficient decreased from 0.0379 to $0.0138 \mathrm{~cm} / \mathrm{s}(63.59 \%)$. Obviously, carbonate precipitation induced clogging within the pores of the sand column (Chu \& Ivanov, 2012). 


\begin{tabular}{ccc}
\hline \multirow{2}{*}{ Grouting round } & \multicolumn{2}{c}{ Parameters } \\
\cline { 2 - 3 } & Flow rate $\left(\mathrm{mL} \cdot \mathrm{min}^{-1}\right)$ & Permeability coefficient $\left(\mathrm{cm} \cdot \mathrm{s}^{-1}\right)$ \\
\hline 1 & 3.70 & 0.0379 \\
2 & 2.48 & 0.0294 \\
& 1.05 & 0.0138 \\
\hline
\end{tabular}

\subsection{Synchrotron radiation micro-CT imaging}

Industrial CT has been widely used to study the microstructure of geomaterials (Ketcham \& Carlson, 2011; Wildenschild \& Sheppard, 2013). However, for substances with small density differences, the imaging effect of industrial CT based on absorption contrast imaging tends to be dim, which is not conducive for discovering minute details. Synchrotron radiation (SR) is a type of electromagnetic radiation emitted by charged particles when accelerated in a curved orbit and features high brilliance, flux, and collimation, owing to its extremely intense and high energy (Zanette et al., 2011). Using an SR light source, micro-CT yields higher imaging resolution than ordinary X-ray sources. Moreover, a phase contrast is supplemented with traditional absorption contrast imaging, which effectively eliminates artifacts (Xiao et al., 2014). In this context, microCT imaging was conducted with a resolution of $6.5 \mu \mathrm{m}$ in the BL13W1 beamline of the Shanghai Synchrotron Radiation Facility (SSRF), an advanced third-generation light source in China. The sample was gently and carefully transferred into the working chamber of BL13W1 and fixed onto a turntable. No disturbance occurred during the transfer and fixation processes. As the microfocus Xray source was fixed, the sample was rotated $360^{\circ}$ along the horizontal direction at a constant speed to obtain perspective images of the entire sample.

Figure 2 illustrates the operating principles of the imaging device. The vertical tomographic interval of the scanning was set to $20 \mu \mathrm{m}$. The sand column was scanned for $120 \mathrm{~min}$ to obtain 11226 tomography scanning images, with 32-bit grayscale in TIFF format. The initial sample was first scanned by SR micro-CT and moved down gently to perform the MICP cementation test to obtain the cemented sample. 


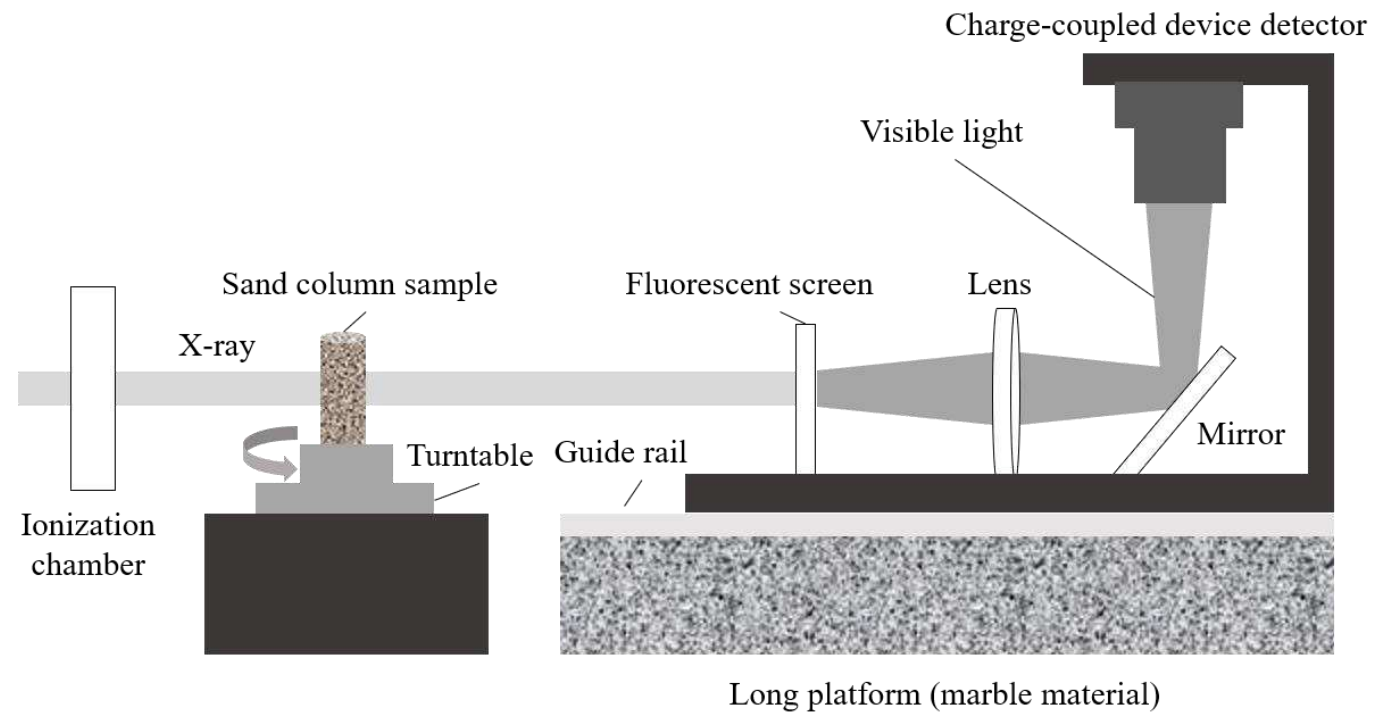

Fig. 2 Operating principle of SR micro-CT imaging

\subsection{Image preprocessing}

The original tomographic sequence images obtained by SR micro-CT need to be preprocessed to improve image clarity, to suppress irrelevant information, and to properly digitalized the image for later quantitative characterization (Xiong et al., 2020). An open-source software, ImageJ, was used to transform the original 32-bit grayscale image into an 8-bit image to efficiently downscale the data space. Subsequently, histogram equalization and smooth filtering were implemented. Figures 3 (a), (b), and (c) show a cross-sectional scanning image of the cemented sample and the subsequent histogram of the equalized and smooth filtered samples, respectively.

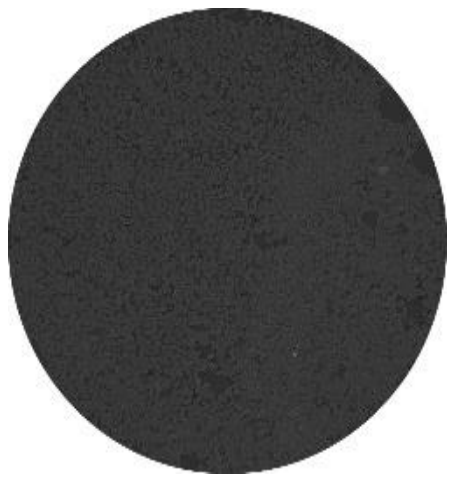

(a) Original image

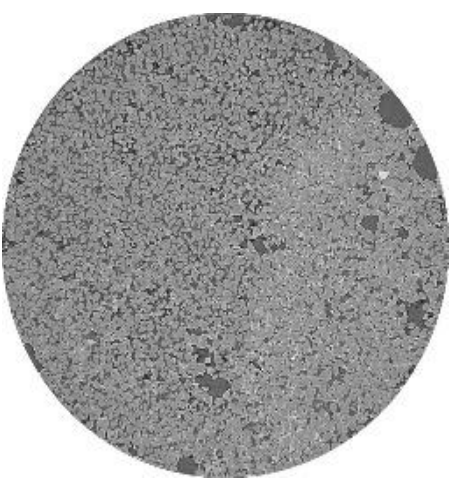

(b) Histogram equalized image

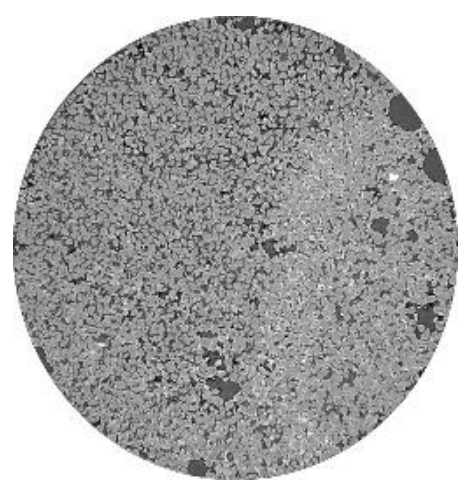

(c) Smooth-filtered image

Fig. 3 Image preprocessing

\subsection{Identifying multi-level thresholds}

Figures 4 (a) and (b) compare the preprocessed images of the initial and cemented samples and their magnification boxes at the same height. The cemented sample seems more compact than the 
initial sample, apart from the occurrence of voids. Specifically, a class of new substances with higher grayscale occurs between the sand particles and pores of the cemented sample. Previous studies confirm that the carbonate precipitation produced by micro-organisms is fixed around sand particles (Muynck et al., 2010; Dejone et al., 2013).

Therefore, two thresholds, $t_{1}^{*}$ and $t_{2}^{*}$, are used for the tri-level segmentation, which satisfies:

$$
\left(t_{1}^{*}, t_{2}^{*}\right)=\arg \max _{0 \leq s_{1}<s_{2} \leq l-1} H\left(t_{1}, t_{2}\right)
$$

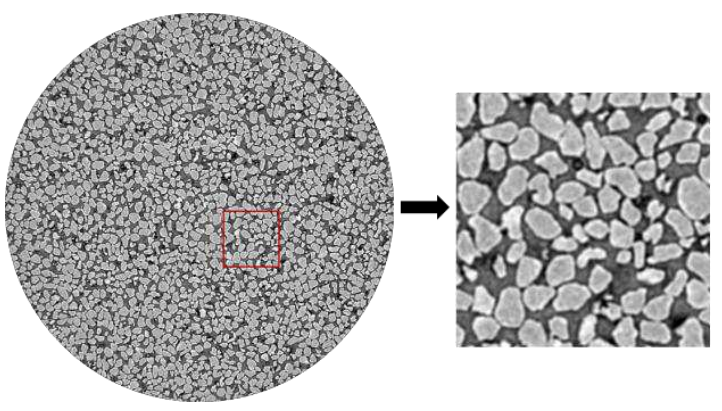

(a)

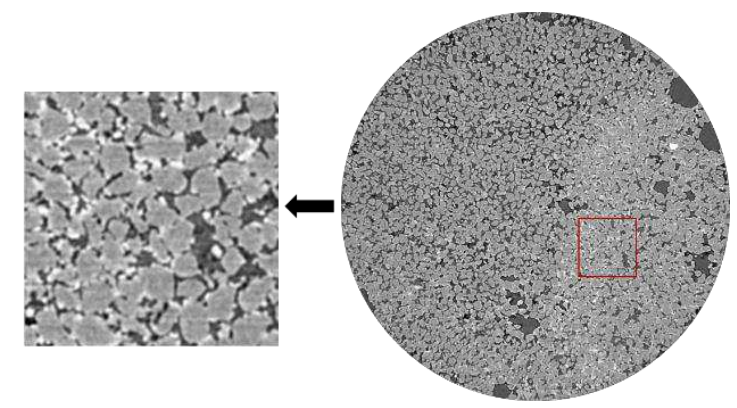

(b)

Fig. 4 Sectional variation after MICP-cementation (a) initial sample; (b) cemented sample.

During scanning, influencing factors (e.g., the instability of light intensity and inhomogeneous spatial distribution of substances) may cause the imaging effect of the transverse section to vary significantly along the axial direction, resulting in threshold variation when performing multi-level segmentation (Cnudde \& Boone, 2013). Therefore, varying thresholding arrays was introduced into the scanning image sequence for more precise segmentation. Specifically, given the circular cross sections of the cylinder sample under scanning, a square image is inscribed for thresholding sampling, which can be easily transformed into a digital matrix; thus, maximal information can be obtained from the ascribed square. Then, KE multi-level algorithm was applied to the square image sequence to identify a thresholding matrix with $m$ rows and 2 columns. The thresholding matrix was applied for the tri-level thresholding of the entire image sequences of both samples. Figure 5 shows the entire process, and Fig. 5 (b) uses part of the image sequence for the demonstration. Such a batch processing strategy is nested into the presented the KE multi-level algorithm algorithm for processing numerous micro-CT scan sequence images. 


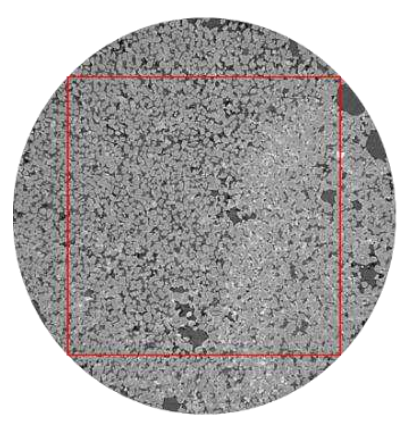

(a)

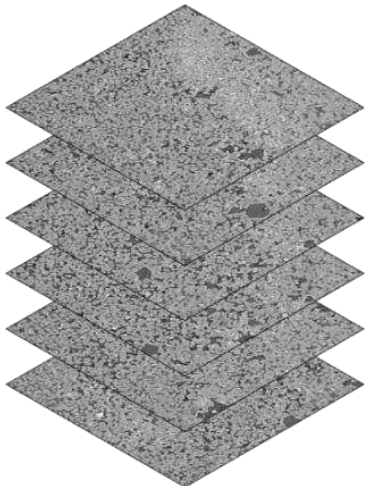

(b)

Fig. 5 Identifying tri-level threshold matrix (a) inscribed square sampling; (b) part of sampled image sequence

\section{Results}

\subsection{Thresholding segmentation}

Watershed and OTSU algorithms were introduced to compare the performance of the KE multilevel algorithm. The watershed algorithm is designed based on the principle of identifying individual geographic catchment area by the downhill flow flowing to the local low point. Previous studies show that the watershed algorithm separates regularly shaped particles in a typical soil specimen. However, a single sand particle may be divided into two or more parts because of the limitation of its algorithm principle (Zheng \& Hryciw, 2016; Sun et al., 2019). The OTSU algorithm (also known as the maximum inter-class variance method) determines the segmentation threshold based on the criterion of maximizing the variance between the target and the background classes. The OTSU algorithm is a robust method owing to its simple principle and convenient implementation, which is suitable for images with low complexity, but it performs poorly for segmenting soil CT images (Otsu, 1979; Houston et al., 2013).

Figure 6 (a) shows the segmentation results of a typical sectional slide image after preprocessing. Segmentation algorithms, including watershed, OTSU, and KE multi-level algorithm algorithms, were used to obtain the segmented image, as shown in Figs. 6 (b-d). The watershed method can only dimly acquires boundaries between particles and pores, let alone effectively distinguish the calcium carbonate crystals. Although the OTSU algorithm can implement tri-level segmentation on the processed image when comparing Figs. 6 (c) and (a), the pixels that should be the original sand particles were inaccurately segmented as carbonate granules. Figure 6 (d) shows that the KE multi-level algorithm algorithm distinctly segments the calcium carbonate crystals from 
the original sand particles and pores and complies well with the three-phase distribution pattern observed in Fig. 6 (a). In addition to the implementation of only two rounds of grouting batches, the volume proportion of calcium carbonate crystals is smaller than that of either sand particles or pores.

The watershed algorithm is based on image morphology and prefers to determine object boundaries without classifying the objects. OTSU prefers the uniformity of the gray level of pixels within the class, making the areas occupied by the object and the background equal. KE identifies the thresholds in accordance with the grayscale in-class probability, which retains tiny objects from the background well.

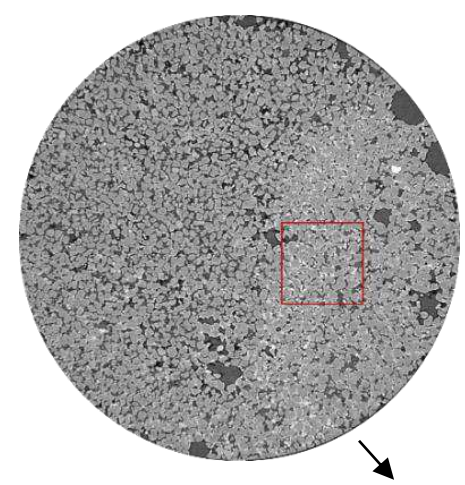

(a)
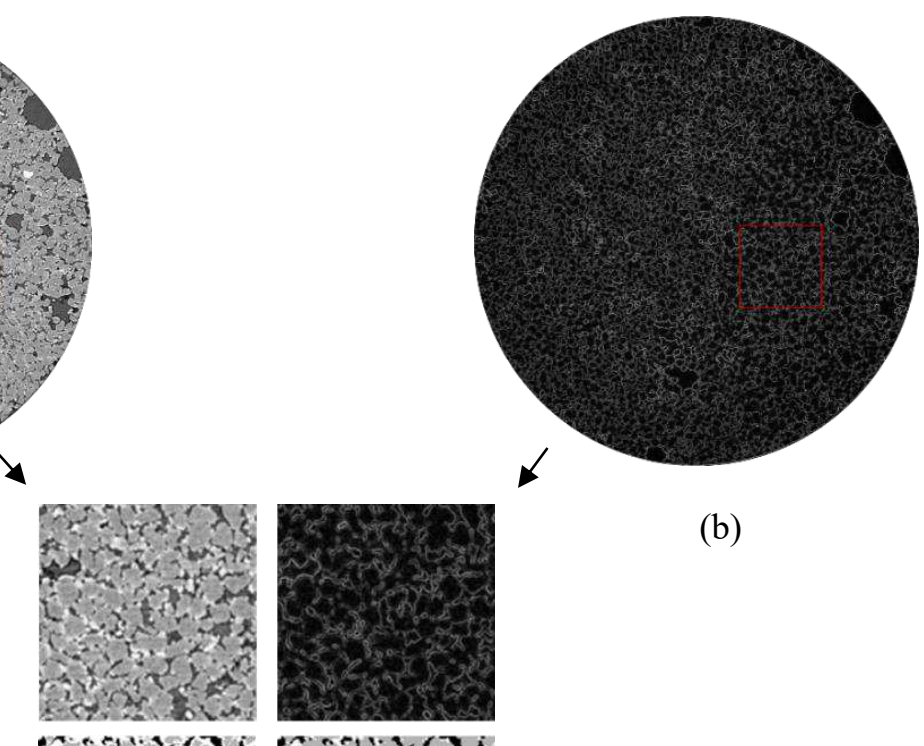

(b)

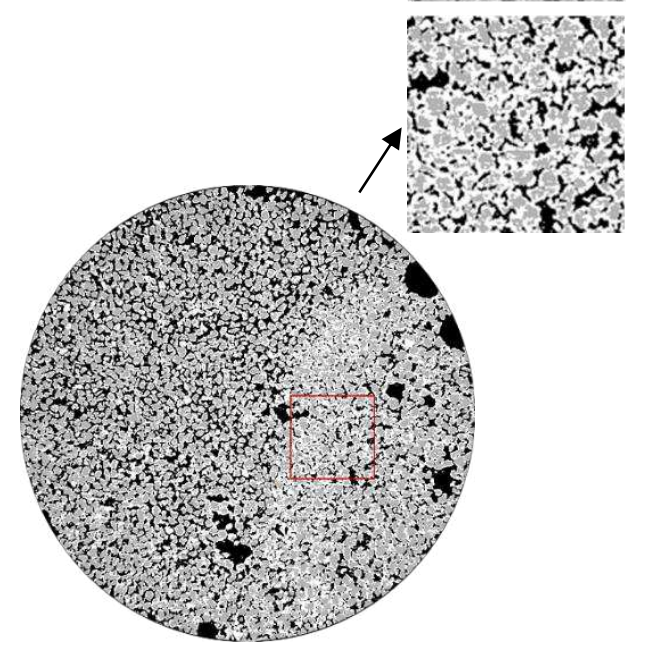

(c)

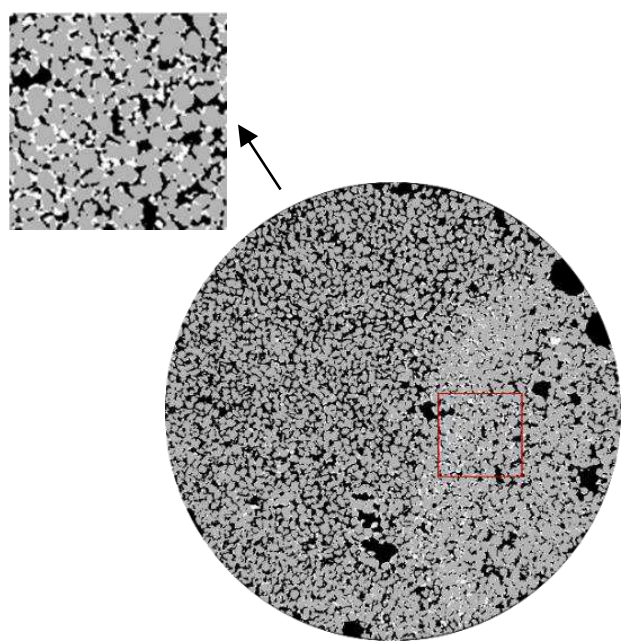

(d)

Fig. 6 Comparison of image segmentation results

(a) Preprocessed image; (b) Watershed; (c) OTSU; (d) KE

Table 2 compares the detailed parameters of the three segmentation algorithms. The watershed does not have a defined threshold value, while OTSU and KE have dual thresholds of 113 and 168, 
and 124 and 202. The running time of KE costs more than that of OTSU but less than that of Watershed. The PSNR and RMSE indices (Bhandari et al., 2016) were used to characterize the intensity and correctness of the segmented images compared with the preprocessed images. KE had maximum PSNR and minimum RMSE; thus, images segmented by KE had the best quality among the three algorithms. In addition, SSIM and FSIM (Wang et al., 2004) were used to represent the similarity of the segmented and preprocessed images. KE had the maximums of SSIM and FSIM and retained the most phase details among the three algorithms.

Table 2 Comparison of quality results of different image segmentation algorithms

\begin{tabular}{cccc}
\hline Items & KE & Watershed & OTSU \\
\hline Threshold & $(124,202)$ & - & $(113,168)$ \\
PSNR & 38.3634 & 17.5455 & 34.2196 \\
RMSE & 37.4533 & 106.0584 & 46.0757 \\
SSIM & 0.7532 & 0.1780 & 0.7450 \\
FSIM & 0.8312 & 0.7189 & 0.8131 \\
\hline
\end{tabular}

* PSNR: peak signal noise ratio; RMSE: Root Mean Square Error; SSIM: Structural Similarity Index Measure; FSIM: Feature Similarity Index Measure.

Based on the tri-level segmentation results in Fig. 6 (d), the extraction of a single class of substance can be realized when merging the pixels of the other two classes by assigning them the same grayscale values of 0 or 255 . Figure 7 (a) shows the extraction results of soil pores and the voids in solid particles. The existence of voids should correspond to the space for tiny sand particles introduced by grouting. Figure 7 (b) shows the distribution of the calcium carbonate crystals within the section. The calcium carbonate crystals are not uniformly distributed but locally gather within a band. Figure 7 (c) shows the merged result of Figs. 7 (a) and (b) by image morphology operation, and the MICP-cementation effect can be noted. Comparing Figs. 7 (a) and (c) reveals that the soil pores tend to be slimmer after carbonate precipitation.
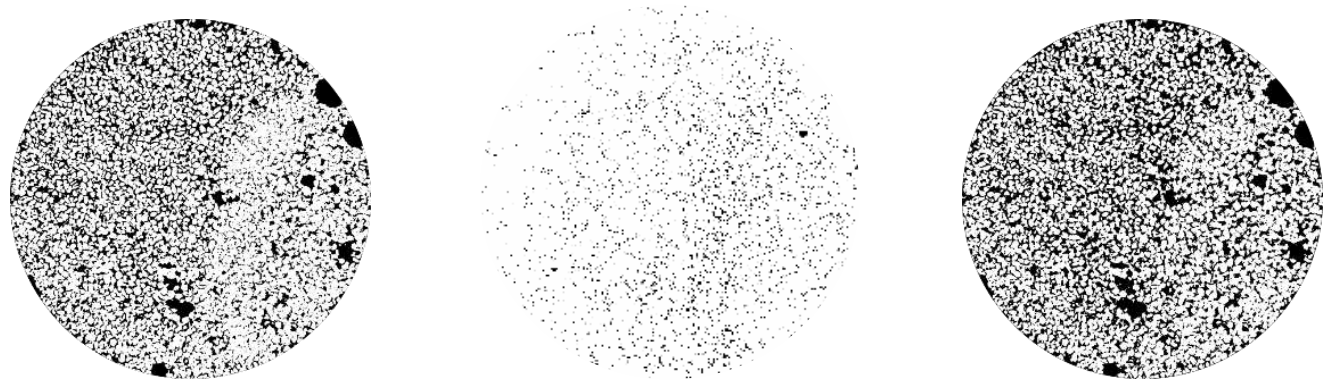
Fig. 7 MICP-cementation effect (a) soil pores and voids (in black); (b) carbonate precipitation granules (in black); algorithms. Figures 8 (a) and (b) show the reconstructed model of the initial and cemented samples, respectively. Multiple voids were distinctly observed at the surface of the cemented model. In addition, the appearance comparison of both models confirms the deductions of the cemented sample in Fig. 4. Considering the numerous sand particles contained in the micro-CT scanning sample, completely characterizing the particles or pores in the sample is difficult owing to the limitations of current computer storage and computing capacity. To simplify the quantitative analysis process and to ensure sufficient representativeness, the detailed 3D profile of both particles and pores and the coordinates of their contact points, can be captured and visualized by a representative elementary volume (REV). A REV is a small volume containing multiple inclusions, over which a measurement can be made to yield values of apparent properties representative of the whole (Bear, 1972). The method is common and well-studied, and determining the unified REV is crucial for studying the microscale structure of sands. We introduced hypothesis testing in a previous study by comparing the convergence of internal structural parameters of REV when different REV sizes were selected. This study uses the inherited method and does not elaborate in detail (Esmaieli et al., 2010; Liu et al., 2019). Figure 8 shows that a group of three cubic REVs at the top, middle, and bottom of the longitudinal axis were extracted from the cemented model. For all the REV cubes, the dimensions were all $300 \times 300 \times 300$ pixels $(1.95 \times 1.95 \times 1.95 \mathrm{~mm})$; the size was determined by statistical analysis. Subsequently, the carbonate precipitation in the three REVs was removed using the above-mentioned method; and the corresponding groups of three new REVs belonging to the comparison model, were obtained. The difference between the two groups of REVs is only the existence of carbonate precipitation. The REVs of the cemented model were consecutively labeled as REV1-1, REV2-1, REV3-1, from the bottom to the top; those of the comparison model were 


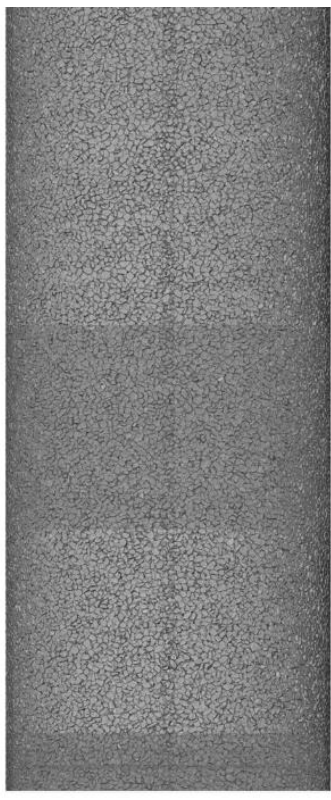

(a)

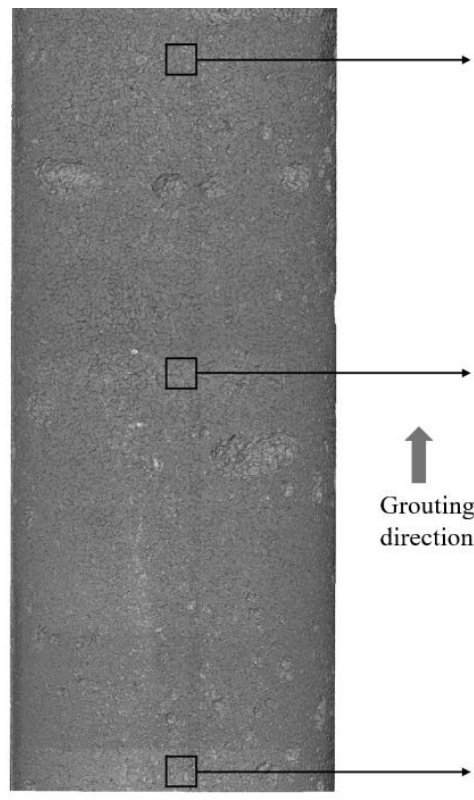

(b)

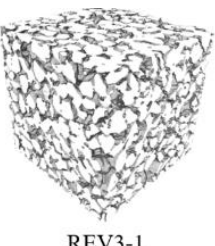

REV3-1

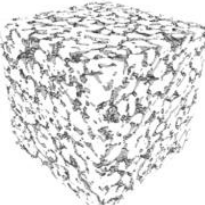

REV2-1

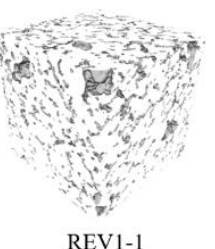

(c)

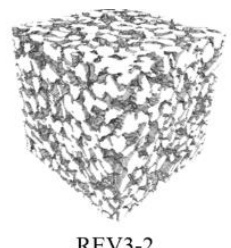

REV3-2

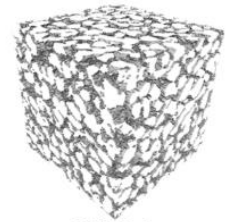

REV2-2

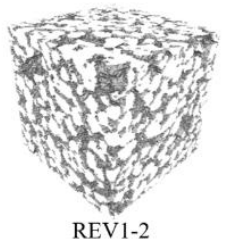

(d)
300 301

302

303

304

305

306

307

308

Fig. 8 Location of REVs (a) 3D reconstruction model of initial sample; (b) 3D reconstruction model of cemented sample; (c) REVs of cemented model. (d) comparison model

The MICP-cementation effect is more intuitively exhibited in Fig. 9. Figure 9 (a) shows the REVs of pores and voids complemented by REV2-1; Fig. 9 (b) shows the REV of all the calcium carbonate crystals in Fig. 9 (a); Fig. 9 (c) shows the REV of the corresponding comparison model in Fig. 9 (a). Comparing Figs. 9 (a) and (c) reveals that the pore connectivity was reduced after the occurrence of carbonate precipitation.

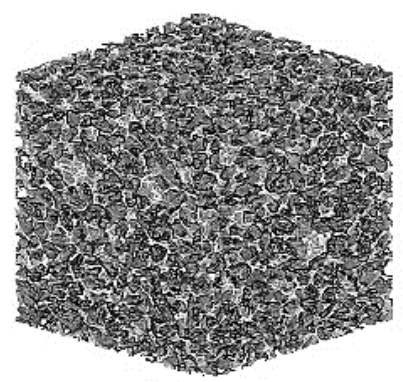

(a)

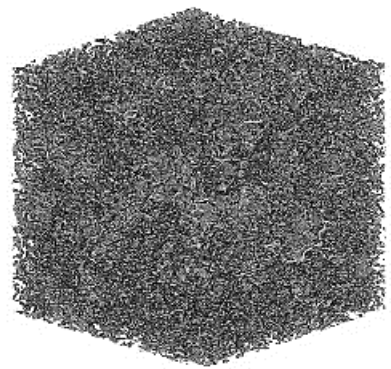

(b)

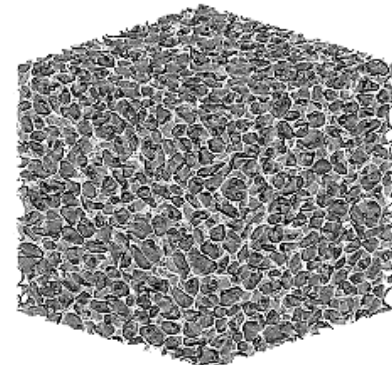

(c)

Fig. 9 Demonstration of MICP-cementation effect (a) cemented model; (b) carbonate precipitation granules; (c) comparison model.

\subsection{Spatial distribution of porosity}

After the thresholding segmentation, the apparent porosity of a single image can be obtained 

porosity $n_{v}$ of the sand column can be approximately denoted by the accumulated apparent porosity values of the discrete tomogram sequence, which yields

$$
n_{v}=\sum_{l}^{u} n_{i}(z) d z
$$

where $l$ and $u$ represent the lower and upper height limits of the entire sample, respectively; $n_{i}(z)$ is the apparent porosity of the binary image at height $z$.

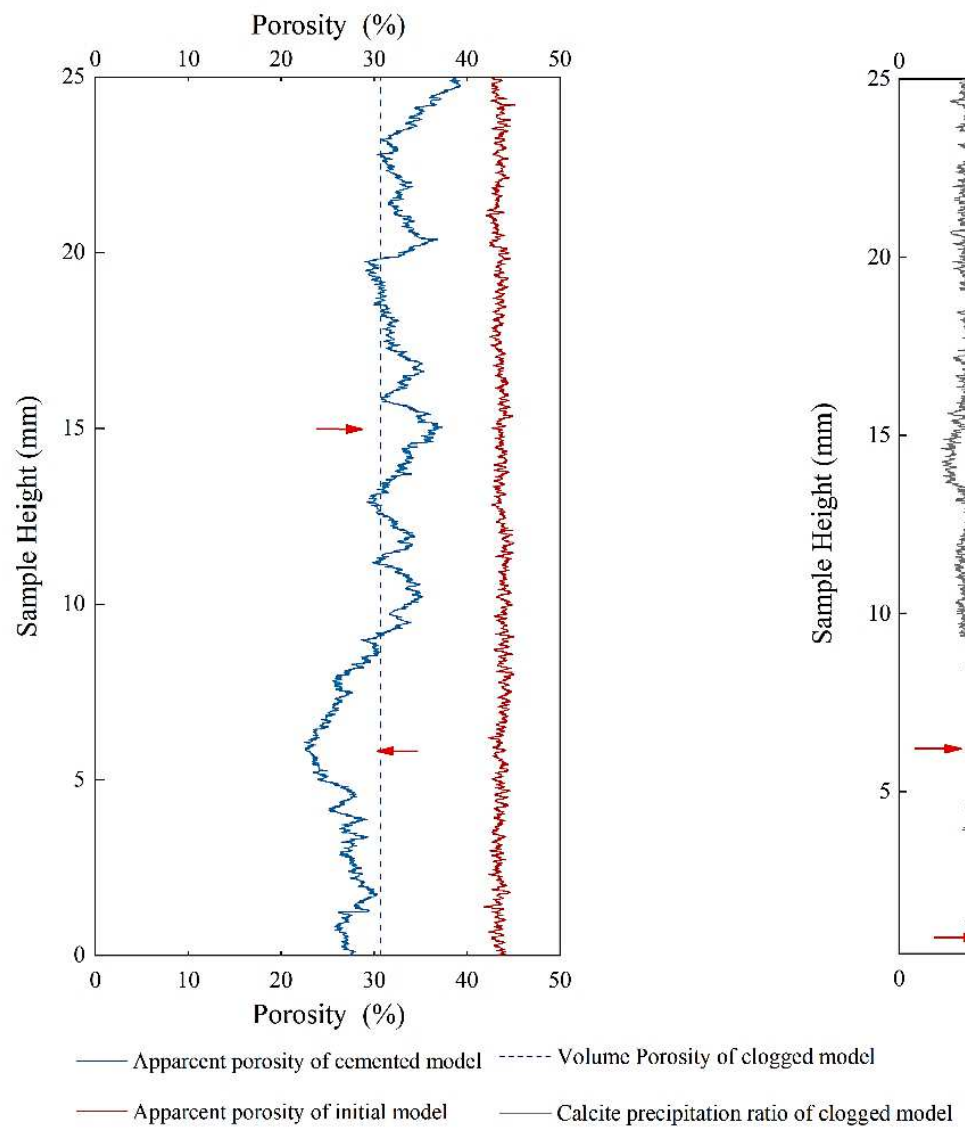

(a)
Pricipitation Content (\%)

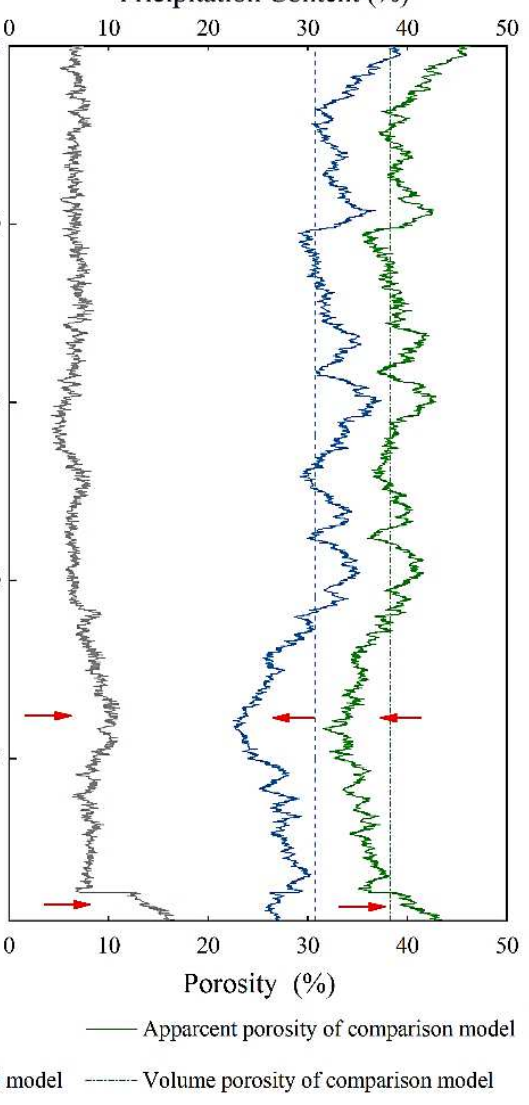

(b)

Fig. 10 Spatial distribution of porosities and carbonate content

Figure 10 (a) compares the porosity of the cemented and initial models; their volume porosities are $30.69 \%$ and $43.62 \%$, respectively. Comparing both volume porosity values reveals a $29.64 \%$ decrease. The apparent porosity of the cemented model increased with fluctuations along the height; the $0-9 \mathrm{~mm}$ part is below $30 \%$, and the rest fluctuates at approximately $30 \%$. The low apparent porosity at $0-9 \mathrm{~mm}$ may be the consequence of the more adequate MICP reaction; the grouting process redistributed the sand particles. The fluctuation of the apparent porosity from 9-25 mm may 
result from the multiple voids of the cemented model, which coincides with the former observation of void existence in Figs. 7 and 8.

Figure 10 (b) shows the apparent porosity of the cemented and comparison models; the content of precipitated carbonate is also indicated. The contents contain distinct variations. At sample heights of $0-2 \mathrm{~mm}$, the apparent porosity of the cemented model varies slightly. However, the porosity of the comparison model varies significantly, primarily because the grouting pressure increases the porosity before precipitation. Furthermore, the content of precipitated carbonate $(12 \%-16 \%)$ at $0-2 \mathrm{~mm}$ was significantly higher than that in the other parts. Therefore, organic matter in the bacterial liquid tends to clog and accumulate at the bottom inlet; the precipitated carbonate accumulates at the inlet, reducing the porosity after precipitation. Specifically, the minimal apparent porosity of the cemented model (approximately $23 \%$ ) occurred at $7 \mathrm{~mm}$, whereas the precipitated carbonate content had a relative maximum of approximately $10 \%$ at the same height. The maximal precipitated carbonate content occurs at $0.5 \mathrm{~mm}$. Thus, the grouting pressure and the gravity of the upper part of the sample make sand particles gather near the 7-mm height region, lowering the porosity of the comparison model. In addition, the content of precipitated carbonate measured by the weighing method was $7.64 \%$, which is close to the average content $(7.58 \%)$ in the reconstruction model based on image segmentation; this demonstrates the accuracy of the proposed algorithm.

\subsection{Pore structure parameters}

As MICP clogs the pores of the soil sample, the parameters of the pore structure characterize the soil structure variation better than the particle group parameters. The maximal ball (MB) algorithm was used to extract the feature parameters of the pore structure from the REVs. MB is an algorithm that statistically analyzes the pore network topology structure of porous media. Maximal inscribed spheres are used to characterize the pore space, hollow cylinders are used to characterize the pore throats, and the coordination number is the number of throats connecting pore bodies (Dong \& Blunt, 2009; Blunt et al., 2013). Figures $11(\mathrm{a}-\mathrm{f})$ show the 3D pore networks of the REVs of both the cemented and comparison models. They were labeled as PREV1-1, PREV2-1, and PREV3-1 for the cemented model and PREV1-2, PREV2-2, and PREV3-2, for the comparison model.

As noted from Fig. 11, the number of large pores and pore throats decreased significantly after MICP cementation. Specifically, the REV1 pore throats were almost eliminated, and large pores 
were mostly transformed to middle and tiny ones without connection. Table 3 contains more details of the comparison between the pore networks of both pore REVs. Eleven soil structure parameters (including porosity, pore number, maximum pore radius, average pore radius, average pore volume, minimum pore volume, average coordination number, average shape factor of the pore area (ASFPA), average pore throat length, median pore throat radius, and average pore throat radius) are analyzed and listed in Table 3. The average shape factor of the pore area is a parameter used to characterize the irregular level of the spatial pores; smaller values denote more irregular the pore space. Quantitatively, the porosity of REV1 decreased from $29.09 \%$ to $9.28 \%$ after cementation; this decreasing trend applies to the porosity of the other two REVs. In addition, the four pore parameters decreased significantly, whereas the followers, pore numbers, and ASFPA increased. The decreasing trend applies to the three pore throat parameters and the average coordinate number; however, the decrease degree is not as high as that of the pores. All these trends imply that the calcium carbonate crystals fill the pore throats and divide an originally large pore into multiple smaller ones; thus, the number of irregular and connected pores decreases.

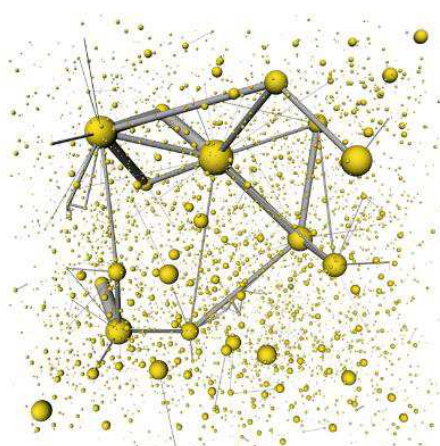

(a) PREV1-1

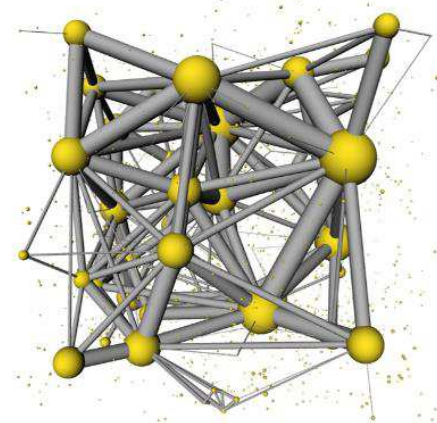

(d) PREV1-2

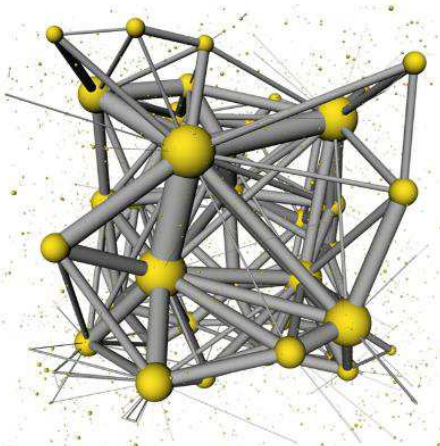

(b) PREV2-1

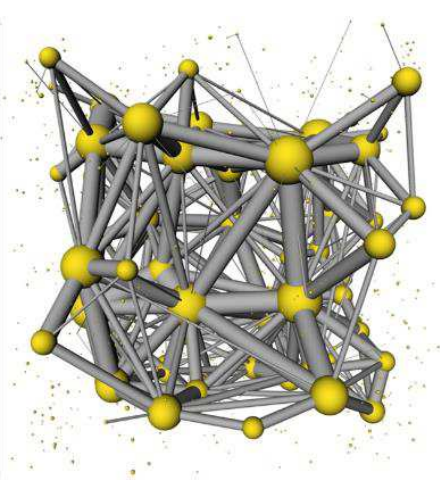

(e) PREV2-2

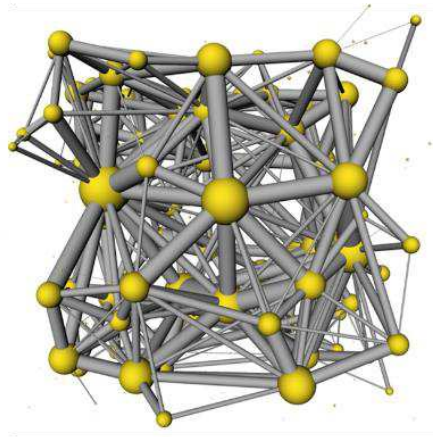

(c) PREV3-1

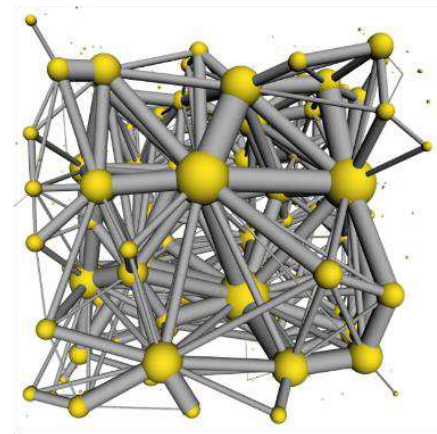

(f) PREV3-2

Fig. 11 Pore REV Networks (PREV1-1, PREV2-1, and PREV3-1 are pore REV networks of the cemented model; PREV1-2, PREV2-2, and PREV3-2 are pore REV networks of the comparison model). 
Table 3 Values of pore structure parameters of the REVs

\begin{tabular}{ccccccc}
\hline Number & PREV1-1 & PREV1-2 & PREV2-1 & PREV2-2 & PREV3-1 & PREV3-2 \\
\hline Porosity & $9.28 \%$ & $29.09 \%$ & $23.95 \%$ & $32.75 \%$ & $34.44 \%$ & $41.48 \%$ \\
Maximum pore radius & 297.93 & 410.50 & 326.08 & 339.34 & 347.63 & 412.174 \\
Average pore radius & 13.11 & 16.09 & 9.36 & 23.52 & 40.91 & 51.43 \\
Average pore volume & 0.12 & 1.21 & 0.48 & 2.60 & 6.01 & 8.06 \\
Minimum pore volume & 274.63 & 2471.62 & 274.63 & 2471.62 & 274.63 & 2471.62 \\
Pore number & 5271 & 1777 & 3000 & 998 & 432 & 409 \\
Average shape factor of & 0.06 & 0.04 & 0.09 & 0.04 & 0.07 & 0.04 \\
Average pore throat & 361.16 & 631.78 & 593.09 & 608.36 & 558.53 & 559.56 \\
Median pore throat & 4.37 & 36.19 & 39.99 & 49.75 & 53.00 & 53.12 \\
Average pore throat & 12.58 & 46.98 & 46.76 & 54.28 & 55.42 & 59.66 \\
Average coordination & 0.06 & 0.26 & 0.16 & 0.76 & 1.86 & 2.20 \\
\hline
\end{tabular}

Furthermore, Fig.12 compares the statistical distributions of the four major pore structure parameters (i.e., coordination number, shape factor of pore area, length, and pore throat radius) and depicts the results as violin plots, which are widely used in data science to illustrate data distribution and probability density. The vertical axis of the violin plot indicates different parameter values, and the width in the horizontal direction represents the occurrence frequency of the parameter at this value. The narrower part of the violin corresponds to a lower frequency of occurrence. The three dotted lines in the violin plot represent the upper quartile, the median, and the lower quartile of the entire data set, respectively, as shown in Fig. 12.

Given the coordinate numbers of the three pairs of PREVs in Fig. 12 (a), the density, maximum, and median values all exhibit decreasing trends after MICP cementation, suggesting a decrease in pore connectivity. As shown in Fig. 12 (b), a significant increase in the PREVs of the cemented models indicates that the pore shapes tend to be more regular after MICP cementation. Figures 12 (c) and (d) show that the length and radius of the pore throat of PREV1, which is located at the bottom of the specimen, decreased significantly after MICP cementation. However, two pairs of upper PREVs do not drop significantly, suggesting that carbonate precipitation growing on the particle surface is not intuitively related to the change effects in the pore roar. In contrast, the shape factor of the pore area does not vary significantly with the specimen height, as shown in Fig. 12 (b). 


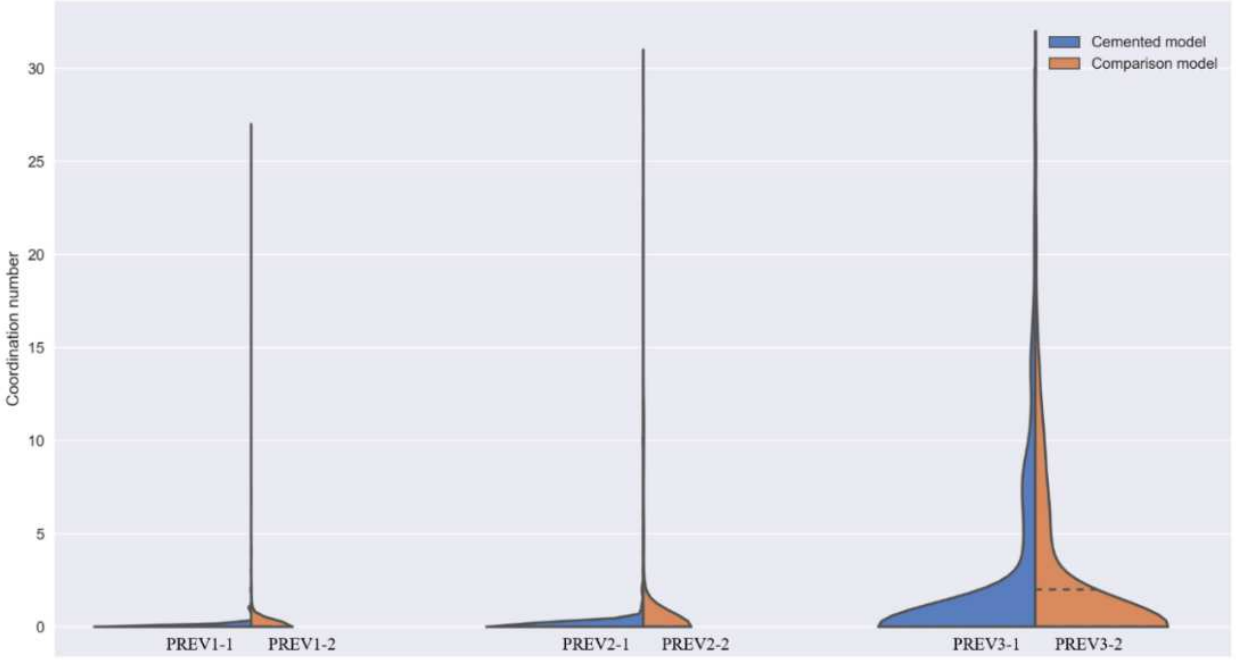

(a) Distribution of the coordination number

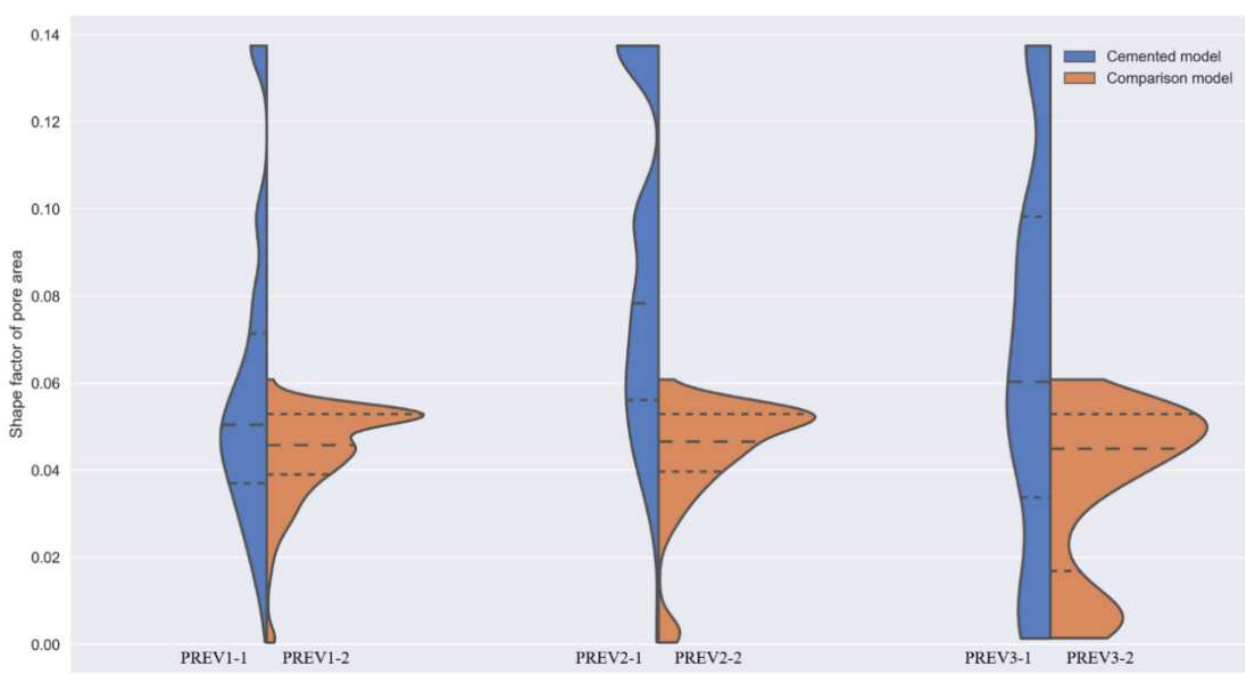

(b) Distribution of pore area shape factors

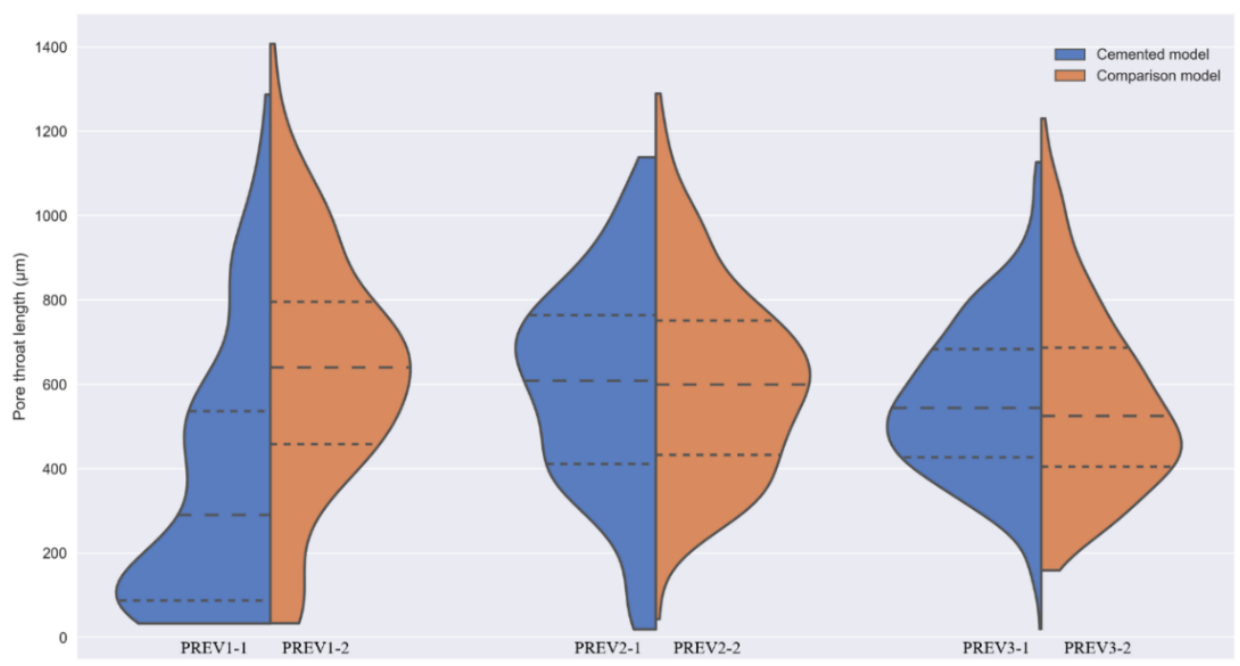

(c) Distribution of pore throat length 


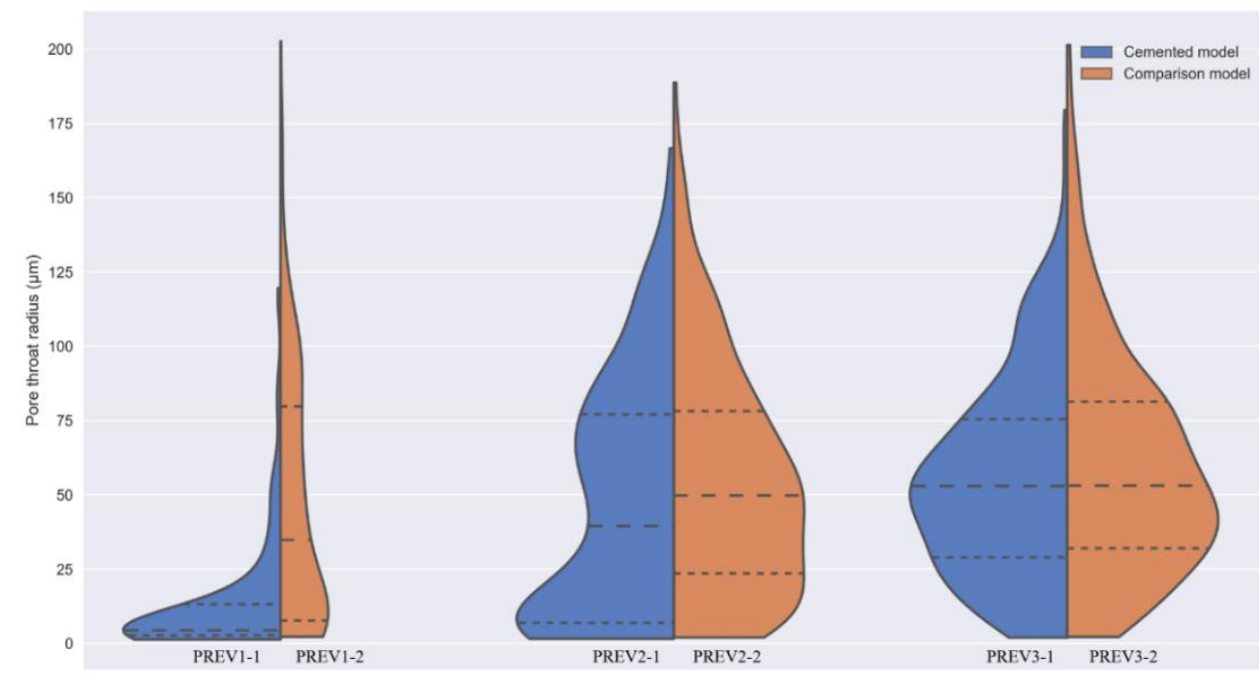

(d) Distribution of pore throat radius

Fig. 12 Comparison of pore structure parameters (in violin plot)

\subsection{Flow characteristics}

The pores of sandy soil offer an open reservoir in which fluid is transported and porosity is primarily determined by the volume and number of pores. Pore throats are relatively long and narrow passages connecting pores and are like capillaries for fluid migration. Pore throat connectivity helps determine permeability (Vogel \& Roth, 2001).

The meso-scale lattice Boltzmann method (LBM) is used for fluid motion simulation. LBM simulates the interaction between the fluid and complex boundary in porous media to finely characterize the flow behavior and to compute macro physical parameters, including permeability. The basic principle of LBM is that the fluid is discretized into a series of fluid particles. The physical region containing the fluid is divided into a series of lattices, and the time is divided into a series of steps; thus, the fluid particles are constrained to move on a limited grid according to certain collision rules. Furthermore, the density, velocity, and other macro parameters can be obtained by statistical analysis of the relevant fluid particle characteristics (Chen \& Doolen, 1998).

The LBM was programmed using MATLAB to simulate the vertical seepage behavior of the six REVs in Fig. 8. The LBGK approximate model and D3Q19 grid discrete mode were employed. D3Q19 is a three-dimensional and nineteen velocity components (Guo et al., 2020). Figure 13 shows the spatial flow fields of the six REVs obtained by the LBM simulation. The flow paths inside a REV are depicted as blue point clouds, and darker point clouds indicate denser flow. The direction 
422 of seepage flow from the bottom to the top corresponds to the test situation. The point cloud color

423 of the corresponding REVs of the cemented model appears lighter than that of the flow fields of the 424 REVs of the comparison model. Thus, the intensity of the flow field, particularly at the bottom of 425 the specimen, is significantly weakened after MICP cementation, which coincides with the pore and pore throat variation trend in Fig. 11 and Table 3.

427

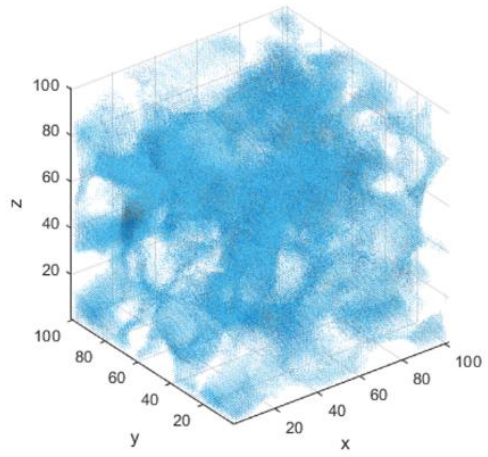

(a) REV3-1

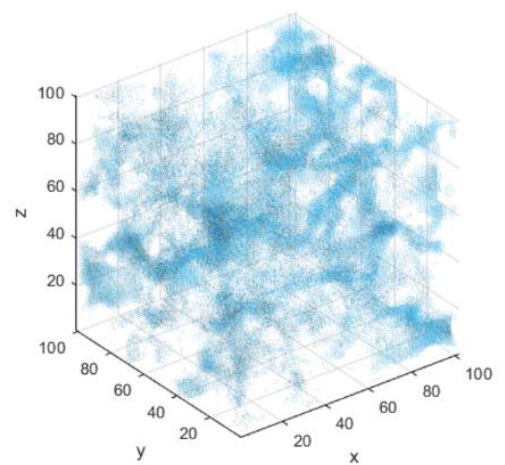

(c) REV2-1

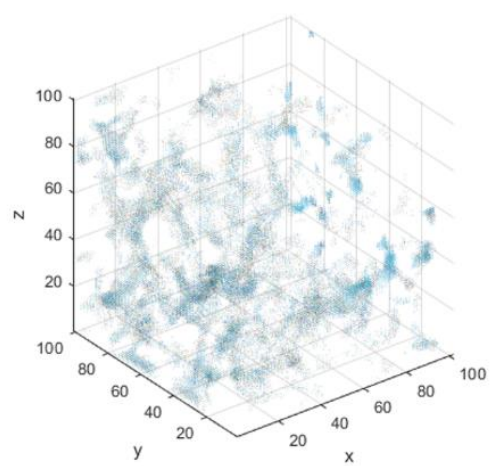

(e) REV1-1

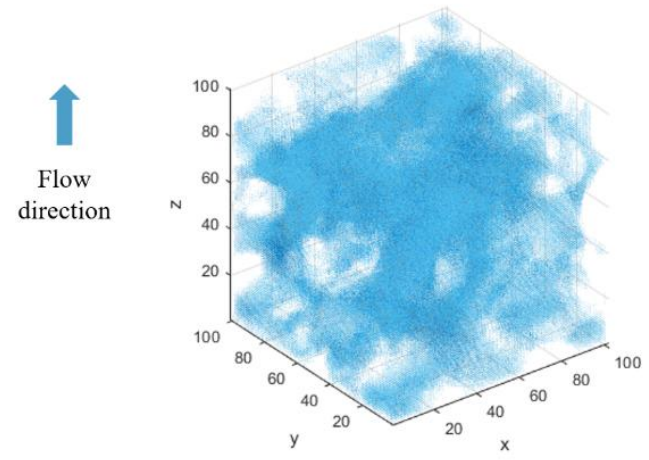

(b) REV3-2

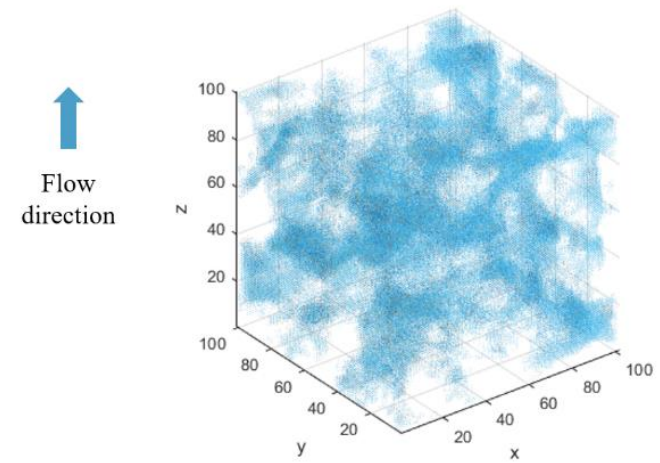

(d) REV2-2

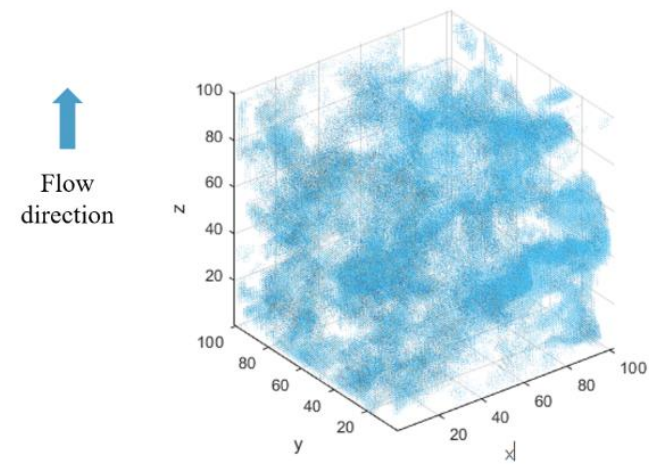

(f) REV1-2

Fig. 13 Comparison of 3D flow field of REVs with vertical seepage

Subsequently, the dimensionless seepage coefficients of the two groups of REVs were 
computed by LBM. From the top to the bottom of the specimen, the seepage coefficients of the cemented and comparison groups were 2.354, 0.986, and 0.351, and 3.317, 1.293 and 1.127. Further, the maximal decrease in the seepage coefficient $(68.86 \%)$ occurred at the bottom of the specimen after MICP cementation; those of the upper and top sections were $29.03 \%$ and $23.74 \%$, respectively, suggesting that MICP reduces the permeability of the sand specimen owing to the clogging impact on the pore structure of the sand column. Compared with the results of the constant head test, only the decrease in the permeability coefficient at the bottom through simulation $(68.86 \%)$ is close to the corresponding result $(63.59 \%)$, the vertical permeability coefficient of the entire MICPcemented sample is controlled by the horizontal section with the greatest degree of clogging.

\section{Discussion}

The inhomogeneity of MICP treated soil is one of the most challenging factors that restricts the up-scale use of MICP in filed application (Tang et al., 2020). Tang et al., (2020) suggest a grouting pressure between 0.1 and 0.3 bar for sand sample, and a grouting speed below $0.042 \mathrm{~mol} / \mathrm{L} / \mathrm{h}$ which can improve the use ratio of cementation solution. Moreover, they point out that excessive grouting pressure would erosion the original soil structure and reduce the solidification effect. In this study, we use a grouting pressure of $0.2 \mathrm{bar}$, and the grouting speeds of $0.012 \mathrm{~mol} / \mathrm{L} / \mathrm{h}$ and $0.0012 \mathrm{~mol} / \mathrm{L} / \mathrm{h}$ for bacterial liquid and cementation solution, respectively. However, soil erosion still occurred and resulted in voids within the specimen in this study, as noted in Fig. 9. This should attribute to the size effect of the specimen. The synchrotron radiation imaging restricts the specimen size to be 10 $\mathrm{mm}$ in diameter and $100 \mathrm{~mm}$ in height, significantly smaller than the specimen size used in previous studies. Thus, a grouting pressure of 0.2 bar could be too high for this specimen to result in erosion inside. Therefore, when considering the factors affecting the performance of MICP treated soil, the size effect should not be ignored. Additionally, note the grouting speed of cementation solution is slow; this is because the calcium carbonate crystals are likely to accumulate around the grouting point to hinder the further injection of cementation solution, which eventually result in the uniformity of calcium carbonate inside the specimen (Joer et al., 2002). Our quantitative characterization method using multi-level thresholding segmentation on synchrotron radiation imaging help to better understand the working mechanism of MICP at the pore scale.

\section{Conclusion}

1) The KE algorithm can efficiently implement multi-level thresholding segmentation on MICP 
treated soils to identify triple components, e.g., pores, sand particles, and calcium carbonate crystals.

2) Computing the apparent porosity and calcium carbonate contents along the specimen height suggested that the grouting pressure enlarged the porosity before precipitation, thus increasing the porosity of the comparison model; in addition, the organic matter is preferentially clogged

\section{Acknowledgement}

The authors would like to thank the anonymous reviewers for their helpful comments and

\section{References}

Arand F, Hesser J. Accurate and efficient maximal ball algorithm for pore network extraction. Comput. Geosci., 101(2017), pp.28-37.

Baveye PC, Laba M, Otten W, Bouckaert L, Sterpaio PD, et al., Observer-dependent variability of the thresholding step in the quantitative analysis of soil images and X-ray microtomography data. Geoderma, 157(2010), pp.51-63.

Bear J. Dynamics of fluids in porous media. New York: American Elsevier, 1972, pp.16-21. 
Bhandari AK, Kumar A, Chaudhary S, Singh GK. A novel color image multilevel thresholdingbased segmentation using nature inspired optimization algorithms. Expert Syst. Appl., 63(2016), pp.112-133.

Blunt MJ, Bijeljic B, Dong H, Gharbi O, Iglauer S, Mostaghimi P, Paluszny A, Pentland C. Porescale imaging and modeling. Adv. Water Resour., 51(2013), pp.197-216.

Chen S, Doolen GD. Lattice Boltzmann Method for Fluid Flows. Annu. Rev. Fluid Mech., 1998, $30(1): 329-364$.

Chu J, Ivanov V. Microbially Induced Calcium Carbonate Precipitation on Surface or in the Bulk of Soil. Geomicrobiol. J., 29(6) (2012), pp.544-549.

Cnudde V \& Boone MN. High-resolution X-ray computed tomography in geosciences: A review of the current technology and applications. Earth-Sci. Rev., 123(2013), pp.1-17.

Dejong JT, Mortensen BM, Martinez BC, Nelson DC. Bio-mediated soil improvement. Ecol. Eng., 36(2) (2010), pp.197-210.

Dejong JT, Soga K, Kavazanjian E, Burns S, Paassen LAV, et al. Biogeochemical processes and geotechnical applications: progress, opportunities and challenges. Geotechnique, 64(4) (2013), pp.287-301.

Dong H \& Blunt MJ. Pore-network extraction from micro-computerized-tomography images. Phys. Rev. E, 80(2009), Article 036307.

Esmaieli K, Hadjigeorgiou J, Grenon M. Estimating geometrical and mechanical REV based on synthetic rock mass models at Brunswick Mine. Int. J. Rock Mech. Min. Sci., 47(6) (2010), pp.915-926.

Frankel RB \& Bazylinski DA. Biologically Induced Mineralization by Bacteria. Biomineralization, 54(2003), pp.95-114.

Guo S, Feng Y, Jacob J, Renard F, Sagaut P. An efficient lattice Boltzmann method for compressible aerodynamics on D3Q19 lattice. J. Comput. Phys., 418(2020), Article 109570.

Hasan A, Alshibli KA. Experimental assessment of 3D particle-to-particle interaction within sheared sand using synchrotron microtomograpgy. Geotechnique, 60(5) (2010), pp.369-379.

Houston AN, Schmidt S, Tarquis AM, Otten W, Baveye PC, Hapca SM. Effect of scanning and image reconstruction settings in X-ray computed microtomography on quality and segmentation of 3D soil images. Geoderma, 207-208 (2013), pp.154-165. 
Iaasonov P, Gebrenegus T, Tuller M. Segmentation of X-ray computed tomography images of porous materials: A crucial step for characterization and quantitative analysis of pore structures. Water Resour. Res., 45(9) (2009), pp.1-12.

Jiang NJ, Tang CS, Hata T, Courcelles B, Dawoud O, Singh DN. Bio-mediated soil improvement: The way forward. Soil Use Manage., 36(2) (2020), pp.185-188.

Joer H, Randolph M, Meritt A (2002) Cementation of porous materials using calcite. Géotechnique $52(5): 313-324$

Kapur JN, Sahoo PK, Wong AKC. A new method for gray-level picture thresholding using the entropy of the histogram. Comput Vis, Graph, Image Proce., 29(3) (1985), pp.273-285.

Ketcham RA \& Carlson WD. Acquisition, optimization and interpretation of X-ray computed tomographic imagery: applications to the geosciences. Comput. Geosci., 27(4) (2011), pp.381400.

Li Z, Liu D, Cai Y, Ranjith PG, Yao Y. Multi-scale quantitative characterization of 3-D pore-fracture networks in bituminous and anthracite coals using FIB-SEM tomography and X-ray $\mu$-CT. Fuel, 209(2017), pp.43-53.

Liu B, Zhu C, Tang CS, Xie YH, Yin LY, Cheng Q, Shi B. Bio-remediation of desiccation cracking in clayey soils through microbially induced Carbonate precipitation (MICP). Eng. Geol., 264(2020), Article 105389.

Liu Y, Zhang W, Liang XL, Xu L, Tang XY. Determination on representative element volume of Nanjing silty-fine sand for its spatial pore structure. Rock Soil Mech.,40(7) (2019), pp.27232729. (In Chinese)

Mitchell JK, Soga K. Fundamentals of soil behavior. third ed. New York, USA: Wiley; 2005.

Muynck WD, Belie ND, Verstraete W. Microbial carbonate precipitation in construction materials: A review. Ecol. Eng., 36(2) (2010), pp.118-136.

Otsu N. A threshold selection method from gray-level histograms. IEEE Trans. Syst. Man. Cybern., 9 (1979), pp. 62-66.

Oda M, Takemura T, Takahashi M. Microstructure in shear band observed by microfocus X-ray computed tomography. Géotechnique, 54(8) (2004), pp.539-542.

Pare S, Kumar A, Bajaj V, Singh GK. A multilevel color image segmentation technique based on cuckoo search algorithm and energy curve. Appl. Soft. Comput., 47(2016), pp.76-102. 
Schropp A, Hoppe R, Patonmmel J, Samberg D, Seiboth F, Stephan S, Wellenreuther G, Falkenberg G, Schroer CG. Hard x-ray scanning microscopy with coherent radiation: beyond the resolution of conventional x-ray microscopes. Appl. Phys. Lett., 100 (25) (2012), Article 253112.

Sezgin M, Sankur B. Survey over image thresholding techniques and quantitative performance evaluation. J. Electron. Imaging, 13(1) (2004), pp.146-168.

Shashank BS, Sharma S, Sowmya S, Latha RA, Meenu PS, Singh DN (2016) State-of-the-art on geotechnical engineering perspective on bio-mediated process. Environ Earth Sci 75 (1): 270.

Sleutel S, Cnudde V, Masschaele B, et al. Comparison of different nano- and micro-focus X-ray computed tomography set-ups for the visualization of the soil microstructure and soil organic matter. Comput. Geosci., 34(8) (2008), pp.931-938.

Sun Q, Zheng J, Li C. Improved watershed analysis for segmenting contacting particles of coarse granular soils in volumetric images. Powder Technol., 356(2019), pp.295-303.

Sutton S. Measurement of Microbial Cells by Optical Density. J. Valid Tech., 17(1) (2011), pp.4649.

Tang CS, Yin LY, Jiang NJ, Zhu C, Zeng H, Li H, Shi B (2020) Factors affecting the performance of microbial-induced carbonate precipitation (MICP) treated soil: a review. Environ Earth Sci 79 (2): 94.

Taylor HF, O'Sullivan C, Sim WW. A new method to identify void constrictions in micro-CT images of sand. Comput Geotech., 69(2015), pp.279-290.

Vogel HJ, Roth K. Quantitative morphology and network representation of soil pore structure. Adv. Water Resour., 24(3) (2001), pp. 233-242.

Voltolini M, Taş N, Wang S, Brodie EL, Ajo-Franklin JB. Quantitative characterization of soil micro-aggregates: New opportunities from sub-micron resolution synchrotron X-ray microtomography. Geoderma, 305(2017), pp.382-393.

Wang W, Kravchenko AN, Smucker AJM, Rivers ML. Comparison of image segmentation methods in simulated 2D and 3D microtomographic images of soil aggregates. Geoderma, 162(2011), pp. $231-241$.

Wang Z, Bovik A, Simoncelli EP. Image quality assessment: from error visibility to structural similarity. IEEE Trans. Image Process., 13(4) (2004), pp.600-612.

Wei T, Fan W, Yuan W, Wei Y, Yu B (2019) Three-dimensional pore network characterization of 

333.

Wei T, Fan W, Yu N, Wei Y. Three-dimensional microstructure characterization of loess based on a serial sectioning technique. Eng. Geol., 261(2019), Article 105265.

Whiffin VS, L.A. van Paassen, Harkes MP. Microbial carbonate precipitation as a soil improvement technique. Geomicrobiol. J., 25(5) (2007), pp.417-423.

Wildenschild D, Sheppard AP. X-ray imaging and analysis techniques for quantifying pore-scale structure and processes in subsurface porous medium systems. Adv. Water Resour., 51(2013), pp.217-246.

Wu Y, Meng T, Wu S. Research Progress of Image Thresholding Methods in Recent 20 Years (19942014). Journal of Data Acquisition \& Processing, 30(1) (2015), pp.1-23. (In Chinese)

Xiao T, Xie H, Deng B, Du G, Chen R. Progresses of X-Ray Imaging Methodology and Its applications at Shanghai Synchrotron Radiation Facility. Acta Optica Sinica, 34(1) (2014), pp. 9-23. (In Chinese)

Xiong QR, Li K, Yang DS, Yu HD, Pan ZJ, Song Y (2020) Characterizing coal pore space by gas adsorption, mercury intrusion, FIB-SEM and $\mu$-CT. Environ Earth Sci 79(5): 209.

Zanette I, Weitkamp T, Lang S, Langer M. Quantitative phase and absorption tomography with an X-ray grating interferometer and synchrotron radiation. Phys. Status Solidi A-Appl. Mat., 208(11) (2011), pp.2526-2532.

Zheng J \& Hryciw RD. Segmentation of contacting soil particles in images by modified watershed analysis. Comput. Geotech., 73(2016), pp.142-152. 